Conclusion As the mental health needs of children and adolescents continue to rise, it is clear there remains an urgent and unmet need for mental health training for clinicians. This data suggests that low-technology high-fidelity simulation is a feasible method of improving clinician confidence in the assessment and management of adolescents with mental health presentations. It is hoped that by developing such programmes, clinicians will be encouraged to cultivate the necessary skills to appropriately assess and manage patients presenting with mental health concerns.

\section{P31 RACISM AND ITS HARMFUL EFFECTS ON NON- DOMINANT RACIAL, ETHNIC YOUTH AND YOUTH- SERVING PROVIDERS: A CALL TO ACTION FOR ORGANIZATIONAL CHANGE}

\begin{abstract}
${ }^{1,2} \mathrm{MV}$ Svetaz ${ }^{*}{ }^{3,4}{ }^{4} \mathrm{~T}$ Coyne-Beasley. ${ }^{1}$ Division of Family and Community Medicine, Hennepin Healthcare System, Minnneapolis, USA; ${ }^{2}$ Leadership Education in Adolescent Health (LEAH) Program, Division of General Pediatrics and Adolescent Health, Minneapolis, USA; ${ }^{3}$ Division Director, UAB Adolescent Medicine, Children's of Alabama, Birmingham, USA; ${ }^{4}$ Past President, Society for Adolescent Health and Medicine, Society for Adolescent Health and Medicine, Chicago, USA
\end{abstract}

\subsection{6/bmjpo-2019-RCPCH-SAHM.36}

Aims Racism can exert negative effects on the self-concepts, health, well-being and life trajectories of both non-dominant racial-ethnic (NDRE) youth and youth-serving providers. In the face of growing nationalism, ethnocentrism, xenophobia and overt expressions of racism, the Society for Adolescent Health and Medicine (SAHM) recognized the critically important need to address the issue of racism and its impact on both NDRE youth and youth-serving providers. SAHM decided to create a guide for organizations involved in clinical care delivery and health professions training and education to promote safe and affirming environments with the goal to create inclusivity during this difficult time.

Methods In developing this position paper, the authors relied on a review of the literature and expert consensus.

Results The Society for Adolescent Health and Medicine endorses the following positions:

- Organizations, providers, researchers, and policymakers should recognize that racism negatively affects the selfconcept, health and well-being, and life trajectories of both NDRE youth and youth-serving providers.

- Organizations should consider and address racism as a form of structural violence.

- Organizations should reaffirm their commitment to justice and equity and actively develop, implement, and evaluate policies and processes to ensure that racism is not embedded systematically.

- Youth-serving organizations should explicitly convey their views against racism and create safe, welcoming spaces for all.

- Organizations should develop, implement, and evaluate interventions at all levels addressing chronic minority stress and vicarious trauma affecting NDRE providers.

- Organizations should develop, implement, and evaluate interventions at all levels addressing chronic minority stress and vicarious trauma affecting NDRE trainees and students.

- Organizations should develop, implement, and evaluate training for providers to routinely explore and address racism with all youth and effectively intervene when they identify affected youth.
- Providers caring for youth should integrate promising interventions to address racism as a part of routine evaluation and in response to identified aggression.

Conclusions In this position paper, SAHM affirms its commitment to foundational moral and ethical principles of justice, equity, and respect for humanity; acknowledges racism in its myriad forms; defines strategies to best promote resiliency and support the health and well-being of NDRE youth, providers, trainees, and students; and provides recommendations on the ways to best effect systemic change.

\section{P33 ANTIBODIES SUGGESTIVE OF IMMUNITY TO HEPATITIS B AND A ARE NOT DETECTED IN THE MAJORITY OF ADOLESCENTS AND YOUNG ADULTS SEEKING MEDICATION-ASSISTED TREATMENT FOR OPIOID USE DISORDER}

${ }^{1,2}$ E McKnight* ${ }^{3}$ S Stull, ${ }^{1,2}$ S Matson, ${ }^{1,2,4} \mathrm{~A}$ Bonny. ${ }^{1}$ Division of Adolescent Medicine Nationwide Children's Hospital, Columbus, USA; ${ }^{2}$ The Ohio State University, Columbus, USA; ${ }^{3}$ Clinical Pharmacology and Therapeutics Research Branch, NIH/NIDA/IRP, Bethesda, USA; ${ }^{4}$ The Research Instutite at Nationwide Children's Hospital, Columbus, USA

\subsection{6/bmjpo-2019-RCPCH-SAHM.37}

Aims Opioid drugs, particularly morphine and heroin, can result in relative immunosuppression, negatively impacting both innate and acquired immunity. Adolescents and young adults (AYA) with history of injection drug use are at high risk for hepatitis infections; therefore, evaluation of their immune status is imperative. The objective of this study was to document hepatitis immune and infection status among AYA seeking medication-assisted treatment (MAT) for severe opioid use disorder.

Methods A retrospective medical record review was conducted of all adolescent and young adults seeking outpatient MAT for severe opioid use disorder from January 1, 2013 to December 31, 2015. Each chart was assessed for hepatitis A, $\mathrm{B}$, and $\mathrm{C}$ serology testing and prior hepatitis immunization status.

Results Among 193 AYA presenting for MAT who had no evidence of prior hepatitis $\mathrm{B}$ infection, mean age was 19.3 (SD 1.66), 114 (59\%) were female, and 184 (95\%) were white non-Hispanic. Mean age at first opioid use was 15.3 (SD 1.85), and mean age at first heroin use was 17.0 (SD 1.87). Injection drug use and prior incarceration were reported by $136(71 \%)$ and 29 (15\%). Hepatitis C antibody was positive in 66 (34\%). Seropositivity for hepatitis B surface antibody (anti-HBs) and hepatitis A immunoglobulin G (anti-HAV) was $62(32 \%)$ and $45(23 \%)$, respectively. Documentation of hepatitis B immunization initiation was evident in 102 (53\%) and completion in 84 (44\%). Hepatitis A immunization initiation and completion was present in 38 (20\%) and $23(12 \%)$, respectively.

Conclusions Despite recommendationsfor routine vaccination, immunity to hepatitis B and A, as assessed by measurement of anti-HBs and anti-HAV, was not evident in the vast majority of AYA seeking MAT. Documentation of appropriate hepatitis immunization was present in a minority of this high risk population. Further study will explore whether seronegativity to hepatitis $A$ and $B$ is reflective of true lack of immunity, nonimmunization status, or immunosuppression from opioid/heroin misuse in this patient population. 